\title{
Sudden death associated with group A streptococcal infection in an 8-year-old girl with undiagnosed hypertrophic cardiomyopathy
}

\author{
Reg Bragonier, Patrick Oades
}

\begin{abstract}
Summary
An 8-year-old girl died suddenly without prior symptoms. Post-mortem examination identified both systemic group A streptococcal infection and hypertrophic cardiomyopathy. She had no history of cardiac symptoms and was not in a high-risk group for sudden death due to hypertrophic cardiomyopathy. We believe the disseminated but asymptomatic group A streptococcal infection precipitated her early death from hypertrophic cardiomyopathy. Sudden unexpected death during systemic infection should be followed by post-mortem examination to look for evidence of hypertrophic cardiomyopathy, as this diagnosis has genetic implications for other family members.
\end{abstract}

Keywords: hypertrophic cardiomyopathy; streptococcal infection; sudden death

Hypertrophic cardiomyopathy (HCM) is inherited as an autosomal dominant trait, although its occurrence is sporadic in a minority of cases. The prevalence is currently thought to be about 2 in 1000 , with an annual death rate of $1 \%$ per annum. ${ }^{1}$ The characteristic pathological features are asymmetrical ventricular hypertrophy and myofibril disarray, due to defective proteins in the cardiac sarcomere. There is considerable variation in both the mode and timing of presentation and the severity of symptoms, which reflects the heterogeneity of gene mutations that cause the disease. Some individuals have very few, if any, symptoms until late in adult life, while others present in adolescence or childhood with exertional dyspnoea, palpitations, a murmur or syncope. Sudden death is a well-recognised complication of HCM, and sometimes occurs without prior symptoms, but it is rare in the first decade of life. ${ }^{2}$ We report a case in which a young girl with undiagnosed HCM died suddenly during an episode of asymptomatic group A streptococcal (GAS) infection and suggest that her death was triggered by this infection.

\section{Case report}

An 8-year-old girl had had no antecedent symptoms prior to collapsing suddenly while playing outdoors. Her father was immediately called and together with a bystander began basic life support measures until an ambulance arrived. She was intubated by the paramedical crew and was discovered to be in ventricular fibrillation. Following asynchronous cardioversion, asystole ensued and she was transferred to hospital receiving endotracheal ventilation and cardiac massage. Further resuscitation in hospital was unsuccessful. Examination at the time gave no clue to the cause of her death. There were no rashes or evidence of injury. She had been well in the past and in particular had had no symptoms suggestive of cardiac disease. She was the only child of unrelated parents and there was no significant family history. At postmortem, histological examination of lung tissue initially demonstrated only capillary congestion with virtually no polymorph inflammatory reaction. However, subsequent Gram staining revealed numerous Gram-positive cocci (figure 1). Nasal and tracheal swabs cultured group A streptococcus as did cerebrospinal fluid, although there were no signs of meningeal inflammation. The heart weighed $124 \mathrm{~g}$ and asymmetrical septal hypertrophy was evident macroscopically. Microscopically there were typical features of HCM with irregular myofibril hypertrophy and disarray, and interstitial fibrosis (figure 2). There were no abnormalities in the conducting system. The conclusion was that she had died as a result of HCM and that GAS infection may have contributed to this.

\section{Discussion}

The presentation of HCM is variable. Contributing factors in the pathophysiology of HCM

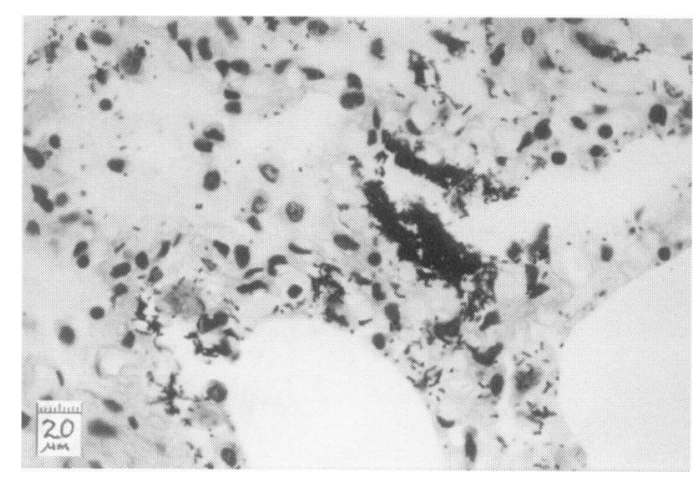

Figure 1 Section of lung tissue showing presence of large numbers of Gram-positive cocci 


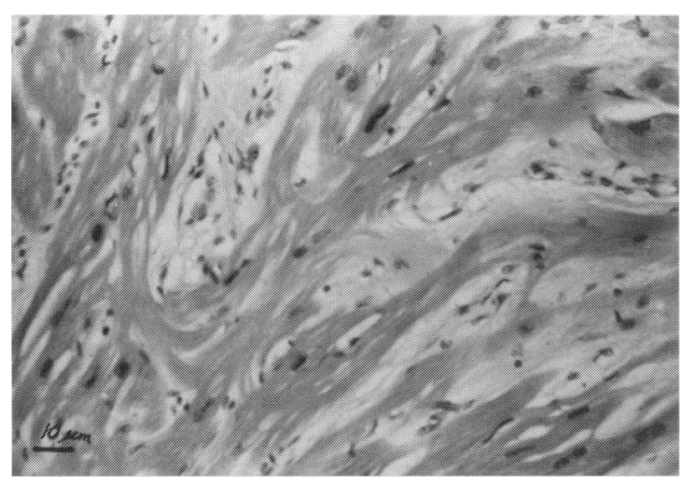

Figure 2 Section of myocardium from the interventricular septum illustrating the characteristic thickened myofibrils in disarray (bundles of fibres streaming in all directions rather than being parallel), interstitial fibrosis and irregular nuclear enlargement with hyperchromasia

include left ventricular diastolic dysfunction, left ventricular outflow tract obstruction, impaired coronary vasodilation, and myocardial ischaemia. ${ }^{2-3}$ These factors may be interrelated and their respective importance will vary between patients. The mechanism of sudden death in patients with HCM is usually cardiac arrest due to tachyarrhythmia. However, both atrial and ventricular tachyarrhythmias are relatively common on ambulatory electrocardiographic monitoring in patients with HCM who remain asymptomatic at the time. ${ }^{2}$ Sudden death in HCM frequently, but not invariably, occurs during or following exercise. The reason for the sudden onset of a fatal arrhythmia in a given patient is unclear but risk factors thought to be important in predicting sudden death are: previous cardiac arrest or sustained ventricular tachycardia, adverse genotype and/or family history of multiple sudden deaths, repetitive or prolonged bursts of ventricular tachycardia on ambulatory electrocardiographic monitoring, recurrent syncope and massive left ventricular hypertrophy. ${ }^{2}$ Treatments aimed at reducing deaths in at-risk patients include left ventricular outflow tract resection (myomectomy), amiodarone therapy, and insertion of a dual chamber pacemaker. ${ }^{13}$ Infection with GAS has a wide variety of clinical manifestations, from relatively benign pharyngitis to headline-provoking soft tissue destruction (necrotising fasciitis). Group A Streptococcal Toxic Shock Syndrome, formally defined in $1993,{ }^{4}$ and described in children, ${ }^{5}$ is perhaps the most dramatic manifestation. It

1 Spirito P, Seidman CE, McKenna WJ, Maron BJ. The management of hypertrophic cardiomyopathy. $N$ Engl $f$ Med 1997;336:75-85.

2 Maron BJ. Hypertrophic cardiomyopathy. Lancet 1997;350: $27-33$

3 Wigle ED, Rakowski H, Kimball BP, Williams WG. Hypertrophic cardiomyopathy - clinical spectrum and treatment. Circulation 1995;92:680-92.

4 The Working Group on Severe Streptococcal Infections. Defining the group A streptococcal toxic shock syndrome. fAMA 1993;269:90-1.

5 Torres-Martinez C, Mahta D, Butt A, Levin M. Streptococcus associated toxic shock. Arch Dis Child 1992;67:126-30.

\section{Summary points}

- HCM is very variable in terms of mode and age of presentation and severity of symptoms

- sudden death from tachyarrhythmia is a well-recognised complication and risk factors have been identified

- sudden death in a young child, even in the face of widespread bacterial infection, requires post-mortem examination in order that this important diagnosis is not missed

comprises evidence of streptococcal infection coupled with rapid onset of shock and multisystem organ failure. The pyrogenic exotoxins released by this organism, termed 'superantigens', have the ability to stimulate T-cells directly, resulting in the massive cytokine cascades which are responsible for the features of toxic shock. ${ }^{6}$ Adults suffering from GAS toxic shock syndrome have been shown to have impaired cardiac performance due to reduced myocardial contractility despite adequate filling pressures and inotropic support. ${ }^{7}$ A direct toxin-mediated cardiac effect has been proposed, supported by the finding that staphylococcal exotoxins (with which GAS exotoxins share similar properties) have been shown to cause myocardial depression in rabbit myocardium in vitro. ${ }^{8}$

The exact role of GAS infection in the death of our patient cannot be certain. $U p$ to the point of her sudden collapse, she had never been troubled by symptoms of cardiac disease, nor did she fall into any of the high risk categories outlined above. It seems likely, therefore, that a fatal arrhythmia was provoked by her intercurrent infection, either by myocardial depressant exotoxins released by GAS or to the resultant cytokine response. This may also explain why she presented with this recognised complication of HCM at such an early age, in the absence of a malignant family history and without prior symptoms. Post-mortem examination in such dramatic cases is essential in order that important diagnoses such as HCM are not missed.

The authors would like to thank consultant pathologists $\mathrm{Dr} T$ Clarke (Royal Devon and Exeter Hospitals) and $\mathrm{Dr} M$ Ashworth (St Michael's Hospital, Bristol) for their help in the preparation of the figures, and consultant microbiologist $\mathrm{Dr} M$ Morgan (Royal Devon and Exeter Hospitals) for her advice and assistance.

6 Holm SE. Invasive group A streptococcal infections. $N$ Engl f Med 1996;335:590-1.

7 Forni AL, Kaplan EL, Schlievert PM, Roberts RB. Clinical and microbiological characteristics of severe group A streptococcus infections and streptococcal toxic shock syndrome. Clin Infect Dis 1995;21:333-40.

8 Olson RD, Stevens DL, Melish ME. Direct effects of purified staphylococcal toxic shock syndrome toxin 1 on myocardial function of isolated rabbit atria. Rev Infect Dis 1989;11(suppl):313-5. 